\title{
Canonical vierbein form of general relativity*
}

\author{
S. Deser ${ }^{\dagger}$ and C. J. Isham \\ Mathematics Department, King's College, London, England WC2R 2LS
}

(Received 17 February 1976)

\begin{abstract}
The Einstein action is expressed in canonical form using the vierbein fields as basic variables. In addition to general covariance, it exhibits the local Lorentz invariance associated with the freedom of choice of the orthonormal frames.
\end{abstract}

\section{INTRODUCTION}

The Lagrangian of general relativity is usually formulated using the components of the metric tensor as the basic field variables. The feasibility of expressing it in canonical for $\mathrm{m}^{1}$ is important when considering the quantization problem even though practical perturbative calculations usually employ covariant, rather than canonical, methods.

Although the metric formulation is appropriate for pure gravity or for gravity plus bosons, the presence of spinors requires the introduction of a larger set of variables. These are the vierbein fields which describe local orthonormal Lorentz frames at each space-time point and with respect to which the spinors are defined. ${ }^{2}$ In order to treat quantum gravity in the general case it is therefore important to obtain a canonical form for the Einstein action using the vierbein fields as basic variables. While the equivalence of the vierbein and metric schemes has been formally established within the covariant path-integral quantization framework, ${ }^{3}$ a complete canonical vierbein formulation has not been exhibited to our knowledge.

In this paper we construct such a formalism directly from the corresponding metric formalism and discuss its properties with particular reference to the freedom of performing local Lorentz rotations. We also consider a "more three dimensional" variant in which only the three-rotation invariance of triads defined on constant-time surfaces is involved. Finally, we comment on the coupled Einstein-Dirac system.

\section{THE VIERBEIN FORMALISM}

We begin with a brief résumé of the definition and properties of vierbein fields. The latter are a set of four orthogonal vector fields $l^{\alpha}(x), \alpha$ $=0,1,2,3$, defined on the space-time manifold. The index $\alpha$ (and, in general, letters from the beginning of the alphabet) labels the independent vectors, each of which also carries a coordinate in$\operatorname{dex} \mu$ in the form $l_{\mu}^{\alpha}(x)$ when expressed in compo- nent form. The noninvertible relation between these sixteen components (the use of vierbein fields as basic variables in the usual second-order form of the action principle does not, of course, yield any information beyond the Einstein equations; one obtains immediately the set $G^{\mu \nu} l_{\nu}^{\alpha}=0$, which when multiplied by the nonsingular quantity $l_{\alpha}^{\rho}$ yields $G^{\mu \rho}=0$ ) and the ten metric components $g_{\mu \nu}(x)$ is embodied in the equations

$$
\begin{aligned}
& l_{\mu}^{\alpha} l_{\nu}^{\beta} \eta_{\alpha \beta}=g_{\mu \nu}, \\
& l_{\mu}^{\alpha} l_{\nu}^{\beta} g^{\mu \nu}=\eta^{\alpha \beta} .
\end{aligned}
$$

We will adopt the usual convention of raising or lowering space-time and "label" $(\alpha, \beta, \ldots)$ indices with the metric tensor and Minkowski metric $\eta_{\alpha \beta}=(+++-)$, respectively. Clearly the verbein fields are only determined by the metric up to an arbitrary "label"-space rotation by the local $\mathrm{O}(3,1)$ Lorentz group. The redundant components of $l_{\mu}^{\alpha}$ specify the relation between the orthonormal frame and the local coordinate system. Corresponding to the 40 components of the metric affinity are the 24 spin connection "rotation coefficients" $B_{\mu \alpha \beta}$ satisfying

$$
\begin{aligned}
& B_{\mu \alpha \beta}=l_{\alpha}^{\sigma} l_{\beta \nu} \Gamma_{\mu \sigma}^{\nu}+l_{\beta \sigma} l_{\alpha, \mu}^{\sigma}, \\
& B_{\mu \alpha \beta}=l_{[\alpha}^{\nu}\left[\partial_{\mu} l_{\nu \beta]}-(\mu \hookleftarrow \nu)-l_{\mu \gamma} l_{\beta]}^{\rho} \partial_{\nu} l_{\rho}^{\gamma}\right],
\end{aligned}
$$

where the square brackets in the subscripts denote antisymmetrization in the indices. Parenthesis will denote symmetrization.

In the metric case the first-order (Palatini) action

$$
I=\int d^{4} x \sqrt{-g}\left[g^{\mu \nu} R_{\mu \nu}(\Gamma)-\Lambda\right]
$$

yields both the Einstein equations and the affinitymetric relation when $g_{\mu \nu}$ and $\Gamma_{\sigma \tau}^{\rho}$ are varied independently. This is a very useful starting point for deriving the canonical form ${ }^{1}$

$$
I=\int d^{4} x\left[\pi^{i j} \dot{g}_{i j}-N_{\mu} R^{\mu}\left(\pi^{l m}, g_{r s}\right)\right]
$$

in terms of the six pairs of conjugate spatial variables $\left(\pi^{i j}, g_{i j}\right) i, j=1,2,3$, the Lagrange multipliers 
$N_{\mu}$ (which are simple functions of $g_{0 \mu}$ ), and the four constraints $R^{\mu}$. The actual calculations leading from (2.5) to (2.6) are rather complicated and we wish to avoid carrying out the vierbein analog [such a procedure has been used successfully by Schwinger ${ }^{6}$ in the triad $\mathrm{SO}(3)$ formalism discussed in the next section]. Instead we shall take advantage of the known metrical results and obtain the desired canonical variables and action directly from them. From (2.1)

$$
g_{i j}=l_{i \alpha} l_{j}^{\alpha} \text { and so } \dot{g}_{i j}=l_{i \alpha} i_{j}^{\alpha}+\dot{l}_{i \alpha} l_{j}^{\alpha},
$$

so that a natural first choice for the vierbein action is

$$
I=\int d^{4} x\left[p^{i \alpha} \dot{i}_{i \alpha}-N_{\mu}\left(l_{\nu \alpha}\right) R^{\mu}(g(l), \pi(p, l))\right]
$$

in terms of the new set of conjugate momenta $p^{i \alpha}$ related to $\pi^{i j}$ by

$$
\pi^{i j}=\frac{1}{4}\left(p^{i \alpha} l_{\alpha}^{j}+p^{j \alpha} l_{\alpha}^{i}\right) .
$$

The constraints are simply rewritten as functions of $p^{i \alpha}$ and $l_{\mu \alpha}$ but it should be emphasized that since the $l_{\alpha}^{j}$ are the space components of the fourdimensional inverse $l_{\alpha}^{\mu}$, the $R^{\mu}$ now depend on both $l_{i \alpha}$ and $l_{0 \alpha}$ as do the $N_{\mu}$ so that (2.6) is not yet the desired form.

We note that the relation in (2.9) is not invertible as $p^{i \alpha}=p^{i \alpha}(\pi)$ in analogy with the impossibility of expressing $l_{\mu \alpha}$ as a unique function of $g_{\mu \nu}$. Indeed, in the present form (2.8) all twelve pairs of "canonical" variables $\left(p^{i \alpha}, l_{j \beta}\right)$ are indisputably present as are the four multipliers $l_{0 \alpha}$. The price for transforming to a redundant set of variables is clearly the need for adding an additional set of six constraints to the action (2.8) since we know that the pure Einstein action can be written in terms of only six pairs of variables (indeed, faced with our final vierbein canonical form, a clever student would notice that six pairs sufficed and might even obtain them explicitly). Theorems on Pfaffian for $\mathrm{ms}^{4}$ can be invoked to show that six constraints are both necessary and sufficient, as can the observation that $(2.8)$ is not $\mathrm{SO}(3,1)$ gauge invariant (because of the $p \dot{i}$ term), whereas (2.6) is trivially so.

The simplest way of constructing the specific form of the new constraints is to insist that the field equations generated by the action integral be equivalent to Einstein's. Their general structure may also be deduced from the $\operatorname{SO}(3,1)$ argument. Indeed, the $p i$ term transforms under local Lorentz rotations

$$
p^{i \alpha} \rightarrow p^{i \beta} \omega_{\beta}^{\alpha}, \quad l_{i \alpha} \rightarrow l_{i \beta} \omega_{\alpha}^{\beta}
$$

as

$$
\left(p^{i \alpha} \dot{i}_{i \alpha}\right) \rightarrow p^{i \alpha} \dot{l}_{i \alpha}+p^{i \beta} l_{i \gamma} \omega_{\beta}^{\alpha} \dot{\omega}_{\alpha}^{\gamma} .
$$

Since $\omega^{\beta}{ }_{\alpha} \omega_{0}{ }^{\alpha}=\delta_{\delta}^{\beta}$, only the skew-symmetric part $\omega_{[\beta}{ }^{\alpha} \dot{\omega}_{\alpha \gamma]}$ contributes, and invariance can be restored if and only if its coefficient vanishes:

$$
J^{[\alpha \beta]} \equiv p^{i[\alpha} \eta_{i}^{\beta]}=0 .
$$

This motivates the addition of a constraint term $m_{\alpha \beta} p^{i \alpha} l_{i}^{\beta}$ to the action with $m_{\alpha \beta}=-m_{\beta \alpha}$ a Lagrange multiplier. Indeed the combination $p^{i \alpha} \dot{i}_{i \alpha}+m_{\alpha \beta} J^{\alpha \beta}$ is $\mathrm{SO}(3,1)$ invariant if $m_{\alpha \beta}$ transforms inhomogeneously as

$$
m_{\alpha \beta}^{\prime}=\omega_{\alpha}^{\gamma} \omega_{\beta}^{\delta} m_{\gamma \delta}-\omega_{\alpha}^{\gamma} \dot{\omega}_{\beta \gamma} .
$$

Such a transformation is natural geometrically since we expect from the $I[l, B]$ form of the action that $m_{\alpha \beta}$ will be related to $B_{0 \alpha \beta}$ which has precisely this property.

The consistency of the action (2.6) when augmented in this way may be verified by demonstrating the closure of the Poisson-bracket algebra generated by the constraint terms $p^{i[\alpha} l_{i}^{\beta]}$ and $R^{\mu}$. Now the action leads to the classical Poissonbracket relations

$$
\left\{l_{i \alpha}(\overrightarrow{\mathrm{x}}), p^{j \beta}(\overrightarrow{\mathrm{y}})\right\}=\delta_{i}^{j} \delta_{\alpha}^{\beta} \delta^{(3)}(\overrightarrow{\mathrm{x}}, \overrightarrow{\mathrm{y}}),
$$

which incidentally imply the correct metric relations

$$
\left\{g_{i j}(\overrightarrow{\mathrm{x}}), \pi^{k l}(\overrightarrow{\mathrm{y}})\right\}=\delta_{i}^{(k} \delta_{j}^{l)} \delta^{(3)}(\overrightarrow{\mathrm{x}}, \overrightarrow{\mathrm{y}}),
$$

while for example

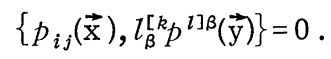

The quantity

$$
\Omega \equiv \int d^{3} x \omega^{\alpha \beta}(x) J_{\alpha \beta}(x), \quad \omega^{\alpha \beta}=-\omega^{\beta \alpha}
$$

may be identified as the generator of "infinitesimal" local Lorentz relations by virtue of the Poisson brackets:

$$
\begin{aligned}
& \left\{l_{i}^{\alpha}(\overrightarrow{\mathrm{x}}), \Omega\right\}=l_{i}^{\beta}(\overrightarrow{\mathrm{x}}) \omega_{\beta}{ }^{\alpha}(\overrightarrow{\mathrm{x}}), \\
& \left\{p^{i \alpha}(\overrightarrow{\mathrm{x}}), \Omega\right\}=p^{i \beta}(\overrightarrow{\mathrm{x}}) \omega_{\beta}{ }^{\alpha}(\overrightarrow{\mathrm{x}}) .
\end{aligned}
$$

The consistency and closure of the $J_{\alpha \beta}$ and $R^{\mu}$ algebra can now be easily checked. Indeed, since the $R^{\mu}$ are $\mathrm{SO}(3,1)$ scalars $\left\{J_{\alpha \beta}, R^{\mu}\right\}=0$ whereas the $\{J, J\}$ relations are just those of the Lorentz algebra:

$$
\left\{J_{\alpha \beta}(\overrightarrow{\mathrm{x}}), J_{\gamma \delta}(\overrightarrow{\mathrm{y}})\right\}=\left[\eta_{\alpha[\delta} J_{\gamma] \beta}(\overrightarrow{\mathrm{x}})-\eta_{\beta[\delta} J_{\gamma] \alpha}(\overrightarrow{\mathrm{x}})\right] \delta^{3}(\overrightarrow{\mathrm{x}}, \overrightarrow{\mathrm{y}}) .
$$

Finally, the closure of the $\left\{R^{\mu}, R^{\nu}\right\}$ relations follows at once from the metric formulation since we have verified that (2.15) still holds.

After this excursion into the local group struc- 
ture we proceed to the field equations. The discussion is greatly simplified by first showing that the four $R^{\mu}=0$ constraints still hold. These should arise by varying the $l_{0}^{\alpha}$, as the latter correspond essentially to $N_{\mu}$. We have

$$
\begin{aligned}
& N_{i} \equiv g_{0 i}=l_{0 \alpha} l_{i}^{\alpha} . \\
& N \equiv N_{i} N_{j}{ }^{(3)} g^{i j}-g_{00}=N_{i} N_{j}{ }^{(3)} g^{i j}-l_{0 \alpha} l_{0}^{\alpha}
\end{aligned}
$$

where ${ }^{(3)} g^{i j}$ is the matrix inverse of $g_{i j}$ and is therefore independent of $l_{0 \alpha}$. Variation with respect to $l_{0 \alpha}$ leads to

$$
\left(\delta N_{\mu} / \delta l_{0 \alpha}\right) R^{\mu}+N_{\mu} \delta R^{\mu} / \delta l_{0 \alpha}=0,
$$

where

$$
\delta N_{i} / \delta l_{0 \alpha}=l_{i}^{\alpha}, \quad N \delta N / \delta l_{0 \alpha}=N_{i}{ }^{(3)} g^{i j} l_{j}^{\alpha}-l_{0}^{\alpha} .
$$

From (2.12) we have in general

$$
\delta l^{\nu \alpha}=-l^{\mu \alpha} l^{\nu \beta} \delta l_{\mu \beta}
$$

and in particular

$$
\delta l^{j \alpha} / \delta l_{0 \beta}=-l^{0 \alpha} l^{j \beta} .
$$

Thus $\delta \pi^{i j} / \delta l_{0 \beta}$ is always proportional to $p^{i \alpha} l_{\alpha}^{0}$. Fortunately, we shall see below that the $J_{\alpha \beta}=0$ constraint (which we have yet to include) implies the vanishing of this quantity. Anticipating this result we drop the $\delta R^{\mu} / \delta l_{0 \alpha}$ terms in (2.23) and, after multiplying by $l_{\alpha}^{\mu}$ and using (2.24), recover the desired equation

$$
R^{\mu}=0 \text {. }
$$

We now turn our attention to the conjugate pairs $\left(l_{i \alpha}, p^{j \beta}\right)$. Varying $p^{i \alpha}$ and using (2.27) gives

$$
\begin{aligned}
i_{i \alpha} & =\delta N_{\mu} R^{\mu} / \delta p^{i \alpha} \\
& =\frac{1}{2} N_{\mu} \delta R^{\mu} / \delta \pi^{i j} l_{\alpha}^{j},
\end{aligned}
$$

since the $R^{\mu}$ depends on $p^{i \alpha}$ only through $\pi^{r s}$. Likewise, varying $l_{i \alpha}$ leads to

$$
\begin{aligned}
-\check{p}^{i \alpha} & =N_{\mu} \delta R^{\mu} / \delta l_{i \alpha} \\
& =\frac{1}{2} N_{\mu}\left(4 l_{s}^{\alpha} \delta R^{\mu} / \delta g_{i s}-p^{r \beta} l^{s \alpha} l_{\beta}^{i} \delta R^{\mu} / \delta \pi^{r s}\right),
\end{aligned}
$$

where (2.25) has been used. Let us now compare (2.28) and (2.29) with the corresponding Einstein equations:

$$
\begin{aligned}
& \dot{g}_{i j}=N_{\mu} \delta R^{\mu} / \delta \pi^{i j}, \\
& -\dot{\pi}^{i j}=N_{\mu} \delta R^{\mu} / \delta g_{i j} .
\end{aligned}
$$

Multiplying (2.28) by $l_{j}^{\alpha}$ (and hence projecting from twelve to nine equations) gives

$$
l_{j}^{\alpha} i_{i \alpha}=\frac{1}{2} N_{\mu} \delta R^{\mu} / \delta \pi^{i j},
$$

the $(i j)$ symmetric part of which is precisely (2.30). Next multiply $(2.29)$ by $l_{\alpha}^{j}$ to obtain the nine equations

$$
\begin{aligned}
-\dot{p}^{i \alpha} l_{\alpha}^{j} & =-\frac{d}{d t}\left(p^{i \alpha} l_{\alpha}^{j}\right)+p^{i \alpha} \dot{l}_{\alpha}^{j} \\
& =2 N_{\mu} \delta R^{\mu} / \delta g_{i j}-\frac{1}{2} N_{\mu} p^{r \beta} l_{\beta}^{i(4)} g^{j s} \delta R^{\mu} / \delta \pi^{r s} .
\end{aligned}
$$

Using the $i_{i \alpha}$ equation, we obtain after some simple algebra

$$
\begin{aligned}
-\frac{d}{d t}\left(p^{i \alpha} l_{\alpha}^{j}\right)= & 2 N_{\mu} \delta R^{\mu} / \delta g_{i j} \\
& +\frac{1}{2} N_{\mu}\left(\delta R^{\mu} / \delta \pi^{r s}\right)\left(p^{i \alpha} l_{\alpha}^{r}-p^{r \alpha} l_{\alpha}^{i}\right)^{(4)} g^{j s} \\
& +\left(p^{i \alpha} l_{\alpha}^{0}\right) l^{j \beta} i_{0 \beta}
\end{aligned}
$$

This equation is unacceptable for two reasons. Firstly, its $(i j)$ symmetric part is not the Einstein equation for $\dot{\pi}^{i j}$ because of the last two terms on the right-hand side. Secondly, it involves the $l_{0 \beta}$ which are not dynamically determined, being essentially Lagrange multipliers like the $N_{\mu}$. Clearly the antisymmetric bracket in (2.34) must be made to vanish (its coefficient is nonsingular) as must the coefficient of $i_{0 \beta}$. Thus the requirement of reproducing the Einstein equations forces these additional constraints on us. One can easily show that the $J_{\alpha \beta}=0$ requirement (2.12) is precisely the correct one. Indeed, multiplying (2.12) by $l_{\beta}^{i} l_{\alpha}^{\mu}$ leads at once to the six desired constraints, namely

$$
p^{i \alpha} l_{\alpha}^{r}-p^{r \alpha} l_{\alpha}^{i}=0, \quad p^{i \alpha} l_{\alpha}^{0}=0 .
$$

It is also necessary to check the consequences of adding the appropriate Lagrange multiplier term

$$
\Delta I=\int m_{\alpha \beta} J^{\alpha \beta} d^{4} x
$$

to the action (2.8). Only the equations of motion $(2.28)$ and (2.29) are affected since $\Delta I$ is independent of $l_{0 \alpha}$ [thus justifying a posteriovi our derivation of (2.27)]. The additional terms on the righthand sides of (2.28) and (2.29) are respectively

$$
\begin{aligned}
& \Delta l_{i \alpha}=-m_{\alpha \beta} l_{i}^{\beta}, \\
& \Delta\left(-\dot{p}^{i \alpha}\right)=m_{\alpha \beta} p^{i \beta} .
\end{aligned}
$$

Correspondingly (2.32) acquires the term $m_{i j}$ $\equiv-l_{j}^{\alpha} l_{i}^{\beta} m_{\alpha \beta}$ which is $(i j)$ antisymmetric, leaving the (already correct) symmetric part unchanged. The skew-symmetric part determines the three projections $m_{i j}$ and, as we remarked earlier, the Lorentz transformation properties of this relation are consistent with Eq. (2.13).

Let us now turn to the modified equations (2.35) in which, by virtue of (2.35), the last two terms 
may be dropped. Two $m$-dependent terms are acquired on the right-hand side: the direct contribution $\Delta_{1}^{i j} \equiv m_{\alpha \beta} l^{j \alpha} p^{i \beta}$ from (2.38) and that due to

$$
\begin{aligned}
\Delta_{2}^{i j} & \equiv-p^{i \alpha} \Delta \dot{l}_{\alpha}^{j}=p^{i \alpha} l^{j \beta} l_{\alpha}^{\mu} \dot{l}_{\mu \beta}=p^{i \alpha} l^{j \beta} l_{\alpha}^{k} l_{k}^{\gamma} m_{\beta \gamma} \\
& =-p^{i \alpha} l^{j \beta} \delta_{\alpha}^{\gamma} m_{\beta \alpha}=-p^{i \alpha} l^{j \beta} m_{\beta \alpha} \equiv-\Delta_{1}^{i j} .
\end{aligned}
$$

Thus the two additional terms cancel, leaving precisely the correct Einstein term on the right-hand side of (2.27) and nothing else. The symmetric part of the left-hand side of (2.34) is just $-\dot{\pi}^{i j}$, while its antisymmetric part $(d / d t)\left(p^{i \alpha} l_{\alpha}^{j}-p^{j \alpha} l_{\alpha}^{i}\right)$ is required to vanish. This, however, is precisely the consistency condition on the first of our constraints (2.35), namely, that if it is initially satisfied it remains so at later times. The consistency of $p^{i \alpha} l_{0 \alpha}=0$ is also explicitly demonstrable:

$$
\frac{d}{d t}\left(p^{i \alpha} l_{\alpha}^{0}\right)=\dot{p}^{i \alpha} l_{\alpha}^{0}+p^{i \alpha} \dot{l}_{\alpha}^{0}
$$

From (2.29) and (2.38)

$$
l_{\alpha}^{0} \dot{p}^{i \alpha}=\frac{1}{2} N_{\mu}\left(\delta R^{\mu} / \delta \pi^{r s}\right) p^{r \beta} l_{\beta}^{i} g^{0 s}-l^{0 \alpha} m_{\alpha \beta} p^{i \beta},
$$

while from (2.28) and (2.37)

$$
\begin{aligned}
l^{00} i_{i \alpha} & =-l_{i \alpha} i^{0 \alpha} \\
& =\frac{1}{2} N_{\mu}\left(\delta R^{\mu} / \delta \pi^{i j}\right) g^{0 s}-l^{0 \alpha} m_{\alpha \beta} l_{i}^{\gamma} .
\end{aligned}
$$

Multiplying (2.42) by $l_{\beta}^{i}$ gives

$$
\begin{aligned}
-\dot{l}_{\beta}^{0}+l_{0 \alpha} l_{\beta}^{0} \dot{l}^{0 \alpha}= & -\dot{l}_{\beta}^{0}-l_{\beta}^{0} l^{0 \alpha} \dot{l}_{0 \alpha} \\
= & \frac{1}{2} N_{\mu}\left(\delta R^{\mu} / \delta \pi^{r s}\right) l_{\beta}^{r} g^{0 s} \\
& -l^{0 \alpha} m_{\alpha \beta}+l^{0 \alpha} p_{0}^{\gamma} l_{\beta}^{0} m_{\alpha \gamma} .
\end{aligned}
$$

Finally, multiplying by $p^{i \beta}$,

$$
p^{i \beta} \dot{l}_{\beta}^{0}=-\frac{1}{2} N_{\mu}\left(\delta R^{\mu} / \delta \pi^{r s}\right) p^{i \beta} l_{\beta}^{r} g^{0 s}+l^{0 \alpha} m_{\alpha \beta} p^{i \beta} .
$$

Adding (2.41) and (2.44) we find that the right-hand sides cancel if the first of Eqs. (2.28) is used to eliminate the $N_{\mu}$ terms. This consistent constraint propagation (the above proof is laborious but instructive; the same conclusions may, of course, be arrived at by looking at the time derivative of the unprojected $J_{\alpha \beta}$ rather than $J_{0 i}$ and $J_{k l}$ separately) is of course just another aspect of the underlying gauge invariance (via the Bianchi identities) or, equivalently, of the algebraic closure of the Poisson brackets of the constraints $\left(J^{\alpha \beta}, R^{\mu}\right)$.

To see that the $m_{\alpha \beta}$ constraints do indeed reduce the action to Einstein form consider the kinetic term:

$$
p^{i \alpha} \dot{l}_{i \alpha} \equiv p^{i \beta} \dot{l}_{i \alpha}\left(l_{\beta}^{\mu} l_{\mu}^{\alpha}\right) .
$$

Decomposing $\mu$ into its space and time components and into symmetric and antisymmetric parts gives

$$
\begin{aligned}
p^{i \alpha} \dot{l}_{i \alpha}= & \frac{1}{4}\left(p^{i \gamma} l_{\gamma}^{m}+p^{m \gamma} l_{\gamma}^{i}\right)\left(i_{i \beta} l_{m}^{\beta}+\dot{l}_{m \beta} l_{i}^{\beta}\right) \\
& +\frac{1}{2}\left(p^{i \gamma} l_{\gamma}^{m}-p^{m \gamma} l_{\gamma}^{i}\right) l_{m}^{\beta} \dot{l}_{i \beta}+\left(p^{i \gamma} l_{\gamma}^{0}\right) l_{0}^{\beta^{\dot{j}}} \dot{l}_{i \beta} \\
= & \pi^{i j} \dot{g}_{i j}
\end{aligned}
$$

on using (2.35). The $m_{\alpha \beta} J^{\alpha \beta}$ term vanishes for $J^{\alpha \beta}=0$ while the $R^{\mu}$ were always functions of $\left(\pi^{i j}, g_{i l}\right)$ only and the $N_{\mu}$ are independent variables. This completes the verification that the action

$$
I=\int d^{4} x\left(p^{i \alpha} i_{i \alpha}-N_{\mu} R^{\mu}+m_{\alpha \beta} p^{i \alpha} l_{i}^{\beta}\right)
$$

is locally $\mathrm{SO}(3,1)$ invariant, that the six new constraints precisely match the redundant pairs of variables and are consistent with the equations of motion, and of course that the field equations are precisely Einstein's. Naturally we could also have introduced six appropriate $\mathrm{SO}(3,1)$ gaugefixing conditions into (2.48) to get the Einstein equations. An intermediate case in which the SO $(3,1)$ symmetry is reduced to $\mathrm{SO}(3)$ by "locking" the timelike vierbein field into world time is interesting in its own right and is treated in the next section. As stated in the Introduction it should also be possible to arrive at (2.47) from first principles using the verbein/spin-connection form of the action with the appropriate geometric components such as $B_{i 0 \alpha}$ and $B_{0 \alpha \beta}$. However, we feel that the technique above is physically clearer, being obtained directly from the already familiar metric form.

\section{TRIADS AND SO(3)}

Our derivation has been carried out in terms of all 16 vierbein components $l_{\mu \alpha}$. Within the spirit of the " $3+1$ " approach of the canonical form (2.4) it might seem more natural to reflect the basic role of the spatial metric $g_{i j}$ by introducing only the 9 triads $e_{i \alpha}(a, b, \ldots=1,2,3)$ satisfying

$$
g_{i j}=l_{i a} l_{j b} \delta^{\alpha b} \text {. }
$$

However, the $g_{0 \mu}$ dependence of $N_{\mu}$ necessitates a hybrid formalism in which the $g_{0 \mu}$ are not decomposed into vierbein fields. In the pure vierbein framework, which is mandatory if fermions are involved, the presence of the $g_{0 \mu}$ requires the introduction of the full set $l_{\mu \alpha}$. The problem then is the relation between the purely spatial $e_{i a}$ and the space-space components $l_{\mu=i, \alpha=a}$. Recalling that

$$
e_{i a} e_{j}^{a}=g_{i j}=l_{i a} l_{j}^{a}-l_{i 0} l_{j 0},
$$


the natural way of accomplishing this is to remove three of the six SO$(3,1)$ degrees of freedom by imposing the gauge conditions

$$
\left(l_{i}\right)_{\alpha=0}=0 \text {, }
$$

leaving just the $\mathrm{SO}(3)$ group of spatial rotations. From Eqs. (2.8) we can see that the Lagrange multipliers take the particularly simple form

$$
N_{i}=e_{i}^{a} l_{0 a}, \quad N=-l_{00},
$$

where the natural square root for $N$ has been taken.

The action now becomes

$$
I=\int d^{4} x\left[p^{i a} \dot{e}_{i a}-\left(l_{0 a} e_{i}^{a} R^{i}-l_{00} R^{0}\right)+m_{[a b]} p^{i a} e_{i}^{b}\right],
$$

in which only the spatial components $m_{a b}$ contribute since $l_{i 0}=0$. The legitimacy of this action ${ }^{5}$ integral may be ascertained precisely as in the $\mathrm{SO}(3,1)$ case. The explicit relations between our $\left(p^{i a}, m_{b c}\right)$ and the rotation coefficients may be computed by comparing Eq. (3.5) with its geometrically derived equivalent. ${ }^{6}$ Thus

$$
m_{b c}=-B_{0 b c}
$$

and

$$
p^{i a}=(\operatorname{det} e)\left(e^{i b} e^{j a}-\frac{1}{2} e^{j b} e^{i a}\right) B_{j 0 b} .
$$

Finally, we note the curious fact that if the symmetric three-scalar density

$$
p^{a b} \equiv p^{i(b} e_{i}^{a)}
$$

is used in $R^{0}$ in place of $\pi^{i j}$, then the quadratic part becomes metric independent:

$$
\pi^{i j} \pi^{l m}\left(g_{i l} g_{j m}-\frac{1}{2} g_{i j} g_{l m}\right)=4\left(p^{a b} p_{a b}-\frac{1}{2} p_{a}^{a} p_{b}^{b}\right) \text {. }
$$

Unfortunately, this is no great advantage since the $R^{i}$ constraints $\pi^{i j}{ }_{1 j}=0$, being a three-vector, cannot be expressed in terms of the $p^{a b}$ alone but read

$$
\left(e_{a}^{i} e_{b}^{j} p^{a b}\right)_{\mid j}=0,
$$

which, since $e_{a \mid j}^{i} \neq 0$, seems no easier to solve than the original form.

\section{THE DIRAC ACTION}

The Dirac action for a spinor coupled to a vierbein gravitational field is

$$
I=\int d^{4} x(\operatorname{det} l)\left[\frac{i}{2} l^{\mu \alpha}\left(\bar{\psi} \gamma_{\alpha} \nabla_{\mu} \psi-\nabla_{\mu} \bar{\psi} \gamma_{\alpha} \psi\right)-m \bar{\psi} \psi\right],
$$

where

$$
\nabla_{\mu} \psi=\left(\partial_{\mu}-\frac{1}{4} i B_{\mu \alpha \beta} \sigma^{\alpha \beta}\right) \psi \text {. }
$$

Here $\gamma^{\alpha}$ and $\sigma^{\beta \gamma} \equiv(i / 2)\left[\gamma^{\beta} \gamma^{\alpha}\right]$ are the usual constant Dirac matrices while $B_{\mu \alpha \beta}[\mathrm{Eq} .(2.4)]$ is the spin connection. As a natural extension of the formalism in the preceeding sections, a canonical form for the coupled Einstein-Dirac system would involve varying $l^{\mu \alpha}$ and $B_{\mu \alpha \beta}$ as independent variables. Weyl ${ }^{7}$ noted long ago that this leads to a slightly different result than that obtained by inserting the geometrical relation between $B$ and $l$ in (4.1) and varying the resulting action with respect to $l_{\mu \alpha}$. The difference, which arises from the derivative nature of the fermion-gravity coupling, manifests itself by the appearance, in the independent $l_{\mu \alpha}, B_{\nu \beta \gamma}$ case, of an additional torsional contribution to the spin connection proportional to $l_{\beta}^{\mu} \bar{\psi} \gamma_{\gamma} \sigma^{\beta \gamma} \psi$. Similar differences arise in electrodynamics when a Pauli term $\bar{\psi} \sigma^{\mu \nu} \psi F_{\mu \nu}$ is introduced. In either case, these extra terms can be compensated if required by adding to the action suitable contact terms essentially proportional to the square of the torsion. Our interest lies not in this aspect but rather in the way in which, in principle, the canonical form is to be achieved for the coupled sys tem. Since we have not carried out the detailed calculations relating $B_{\mu \alpha \beta}$ to $\left(p^{i \alpha}, m_{\alpha \beta}\right)$ we cannot write down the canonical Dirac action explicitly [ such a calculation was successfully performed by $\mathrm{Kibble}^{8}$ using the time-gauge condition with a residual $\mathrm{SO}(3)$ invariance], but there is clearly no obstacle (other than the tedious nature of the calculations) to constructing such a form. Firstly, the metric dependence in the $\partial_{0} \psi$ terms must be removed by absorbing (dete) $\gamma^{\alpha} l_{\alpha}^{0}$ into the conjugate of $\psi$.

The remaining terms will depend on the product of $l_{\mu \alpha}$ and the spatial gradients of the spinors, and on nonderivative terms involving $p^{i \alpha}$ and $m_{\alpha \beta}$ linearly as well as terms involving spatial derivatives of the vierbein fields. The combined sys tems' constraints will include extra contributions to $J_{\alpha \beta}$ and $R^{\mu}$ and the $p^{i \alpha}-\dot{l}_{i \alpha}$ relation will be altered by a torsional term. This situation is for mally not dissimilar to that holding for higher integer $(>1)$ spin fields in the metric formulation, where derivative coupling is also necessarily present, although in the present case the absence of matter-field constraints removes the serious problems present in the former.

\footnotetext{
*Work supported in part by SRC Research Grant No. B/RG/68807, by NATO (a Senior Science Fellowship and Grant No. 815) and by NSF.
}

†On leave from Brandeis University, Waltham, Masschusetts 02154 .

${ }^{1}$ R. Arnowitt, S. Deser, and C. W. Misner, Phys. Rev. 
117, 1595 (1960)

${ }^{2}$ H. Weyl, Z. Phys. 56, 330 (1929); T. W. B. Kibble, J. Math. Phys. 2, 212 (1961).

${ }^{3}$ S. Deser and $\bar{P}$. van Nieuwenhuizen, Phys. Rev. D $\underline{10}$, 411 (1974).
${ }^{4}$ P. Libermann, Bull. Acad. R. Med. Belg. 39, 846 (1953). ${ }^{5}$ C. J. Isham, Proc. R. Soc. London (to be published).

${ }^{6} \mathrm{~J}$. Schwinger, Phys. Rev. 130, 1253 (1963).

${ }^{7}$ H. Weyl, Phys. Rev. 77, 699 (1950).

${ }^{8}$ T. W. B. Kibble, J. Math. Phys. $\underline{4}, 1433$ (1963). 